\title{
The Inhibition Effect of 2-amino-4-chlorobenzothiazole on X65 Steel Corrosion in $\mathrm{H}_{2} \mathrm{SO}_{4}$ Solution
}

\author{
Shujun Chen ${ }^{1, *}$, Siyi Chen ${ }^{1}$, Huajun Zhao ${ }^{1}$, Hao Wang ${ }^{1}$, Pushan Wen ${ }^{1}$, Hao Li $^{2}$ \\ ${ }^{1}$ School of Chemistry and Chemical Engineering, Zunyi Normal University, Zunyi 563006, China \\ ${ }^{2}$ School of Chemistry and Chemical Engineering, Chongqing University, Chongqing 400044, China \\ *E-mail: shujunchen_znu@163.com
}

doi: $10.20964 / 2020.06 .37$

Received: 15 January 2020 / Accepted: 12 March 2020 / Published: 10 May 2020

\begin{abstract}
In this study, we study 2-amino-4-chlorobenzothiazole (ACBT) as a corrosion inhibitor for X65 steel in $\mathrm{H}_{2} \mathrm{SO}_{4}$ medium. The corrosion inhibition performance of ACBT for X65 steel has been studied using electrochemical methods, surface morphology analysis and theoretical calculations. Electrochemical experiment data indicated ACBT belongs to mixed-type corrosion inhibitor. When the concentration of ACBT is $5 \mathrm{mM}$ at $298 \mathrm{~K}$, its corrosion inhibition efficiency is close to $90 \%$. The potentiodynamic polarization curve shows that the inhibition effect of ACBT on the cathodic reaction is significantly greater than that of the anodic reaction. SEM morphology analysis strongly proves the corrosion inhibition performance of ACBT. Quantum chemical calculations and molecular dynamics simulations have revealed the corrosion inhibition properties of ACBT. The adsorption of ACBT on the surface of X65 steel conforms to the Langmuir adsorption isothermal model.
\end{abstract}

Keywords: Corrosion inhibitor; X65 steel; potentiodynamic polarization curve; Quantum chemical calculations; Langmuir adsorption.

\section{FULL TEXT}

(C) 2020 The Authors. Published by ESG (www.electrochemsci.org). This article is an open access article distributed under the terms and conditions of the Creative Commons Attribution license (http://creativecommons.org/licenses/by/4.0/). 